\title{
Nuclear medicine: a global perspective
}

\author{
Diana Paez ${ }^{1} \cdot$ Francesco Giammarile $^{1} \cdot$ Pilar Orellana $^{1}$
}

Published online: 25 March 2020

(C) Italian Association of Nuclear Medicine and Molecular Imaging 2020

In this era of globalization, health systems, primarily in lowand middle-income countries (LMICs) [1] are required to accommodate the care and management of the increasing burden of non-communicable diseases (NCDs). Vast regions of the world also must contend with the double burden of disease, including facing outbreaks of novel viruses. Health systems need to address the epidemiological transition from communicable to NCDs with strengthened capacity. Nuclear Medicine has become an integral part of an efficient health system that acknowledges this shift with the management of various NCDs, in particular cardiovascular, oncological, and neurodegenerative diseases.

Without a doubt, innovation in research and development is a driving force in nuclear medicine. New devices, radiopharmaceuticals, clinical applications, and evidence-based medicine are produced at a fast pace and need to be propagated. New standards of best practices should be emphasized not only as part of training programmes, but also all efforts should be made to keep the medical community abreast with developments to provide optimal patient care and professional growth.

Global health realities show large inequities in access to nuclear medicine services. These are now showcased in an unprecedented, comprehensive manner in the NUclear mEdicine DAtaBase (NUMDAB) [2], as well as in the IAEA Medical imAGIng and Nuclear mEdicine global resources database (IMAGINE) [3].

According to the data available at International Atomic Energy Agency (IAEA) [2, 3], 134 out of 195 countries have nuclear medicine facilities. There are over 25,000 SPECT cameras. The heterogenous global distribution of SPECT systems range between 0.036 cameras/million inhabitants in low-income countries (LIC) to 17.9 cameras/million in

Francesco Giammarile

f.giammarile@iaea.org

1 Nuclear Medicine and Diagnostic Imaging Section, Division of Human Health, International Atomic Energy Agency, Vienna International Centre, PO Box 100, 1400 Vienna, Austria high-income countries (HIC). It should also be noted that the difference between upper middle-income countries (UMIC) and lower middle-income (LMIC) countries is sixfold (1.33 cameras/million vs 0.20 cameras/million, respectively) (Table 1). The regional distribution is also heterogenous. In the Americas, the USA has the highest rate of SPECT cameras/million inhabitants (45.3), whereas Canada has 16 cameras/million. There are 1456 cameras, with a rate of 3.73 cameras/million in Latin America and the Caribbean (LAC). Ranging from Argentina with 8.74 cameras/million, Brazil with 6.36 , Colombia with 2.8 cameras/million inhabitants, Ecuador with 0.7 scanners/million to countries such as Aruba or Barbados with no availability of SPECT scanners.

Inequities in access to PET-CT are more striking. In high-income countries, there are 3.2 scanners/million inhabitants and in low-income settings, 0.007 scanners/million (Table 1).

Regionally, using the African continent as an example, only 9 out of 54 countries have PET scanners.

Stimulating market projections to inject investment and research and development is key to maintaining and growing nuclear medicine access. Currently, the discipline of diagnostic nuclear medicine (SPECT and PET) has the smallest share of the global medical imaging market (including CT, MRI, US, and X-ray) at 6.5\%. It is expected to be the fastest growing segment due to the rising prevalence of NCDs, increased needs for early and accurate diagnoses, new technological developments both in hardware and software, the availability of new tracers, and its welcomed reception in emerging markets.

The LAC region serves to illustrate the global nuclear medicine growth. The practice of nuclear medicine in the region has experienced an important development in the last decades. However, there is great heterogeneity among countries regarding the availability of technology and human resources. According to data collected by the IAEA throughout the years, PET scanners in the LAC region had a Compound Annual Growth Rate of approximately $21 \%$ and grew from 22 systems in 6 countries (out of the 33 countries in the region) in 2005, to 144 systems in 11 countries in 2015, and 
Table 1 Number of scanners per million inhabitants

\begin{tabular}{lcl}
\hline Income group & SPECT & PET \\
\hline HIC & 16.7 & 3.12 \\
UMIC & 1.7 & 0.32 \\
LMIC & 0.20 & 0.11 \\
LIC & 0.04 & 0.007 \\
\hline
\end{tabular}

301 systems in 17 countries in 2019. Although the growth was higher than the global nuclear medicine imaging devices market, the number of PET scanners per million inhabitants is around 0.47. In the Middle East (ME), the number of PET scanners in 2016 was 194 [4] and grew to 220 scanners in 2019, the number of PET scanners per million inhabitants in the ME is 0.66 [3]. In both cases LAC and the ME, the number of PET scanners/million inhabitants is still far below the recommended 2.0 to 2.5 scanners per million in an optimal health setting [5]. As for the SPECT market, there existed 1200 systems in 2015 [6] in 20 out of the 33 LAC countries to 1456 systems in 2019, distributed in 23 countries [3].

In this era of unprecedented scientific and technological developments there is an increasing demand for "a personalized medicine approach" which is the development of better strategies for detecting and treating diseases based on an individual's unique profile. The growth of personalized medicine is propelled by several factors that include the advances in molecular biology, genetics and proteomics, the better understanding of normal and pathological processes, the greater knowledge of the mechanism of individual diseases, the superior identification of disease subtypes and the better prediction of individual patient's responses to treatment.

Expanded use of nuclear medicine techniques has the potential to accelerate, simplify, and reduce the costs of developing and delivering improved health care, reduce the healthcare expenditure and could facilitate the implementation of personalized medicine. Its applications can go far beyond diagnostics, allowing support in the selection of the appropriate therapy, evaluating the therapy response and follow-up; and driving the journey to personalized medicine.

Nuclear medicine has come to a fork in the road, with the choice being whether to remain a diagnostic lesion-detection technique, or whether to join the revolution in precision medicine, characterizing tumour biology and directing treatment through highly specific tracers that guide us to the world of theragnostic with translation molecular imaging being the central focus (Fig. 1).

To reach the full potential of nuclear medicine, including theragnostic, it is essential to train nuclear medicine professionals, not only newcomers but also experienced professionals trained in the field; to work with regulatory bodies to ensure and comply with safety requirements; to cooperate alongside policy makers, to include reimbursements for established and emerging applications, and to implement quality management systems in clinical practice to ensure the safety and quality of services provided.

Nuclear medicine and medical imaging should be included in the main global public policy guidelines for management of patients. Multisectoral coordination amongst key stakeholders (World Health Organization, IAEA, and other United Nations organizations; governmental bodies, professional organizations, non-governmental organizations, and private sector) is crucial to be able to deliver the best care possible. Unfortunately, this multisectoral coordination is currently unclear.

There are some ongoing efforts by the international community to deal with the burden of diseases. In 2015, the United Nations established the Sustainable Development Goals, a set of 17 specific goals aimed at transforming our world by 2030 and achieving a better and more sustainable
Fig. 1 Nuclear medicine in disease management

\section{Nuclear Medicine in Disease Management}

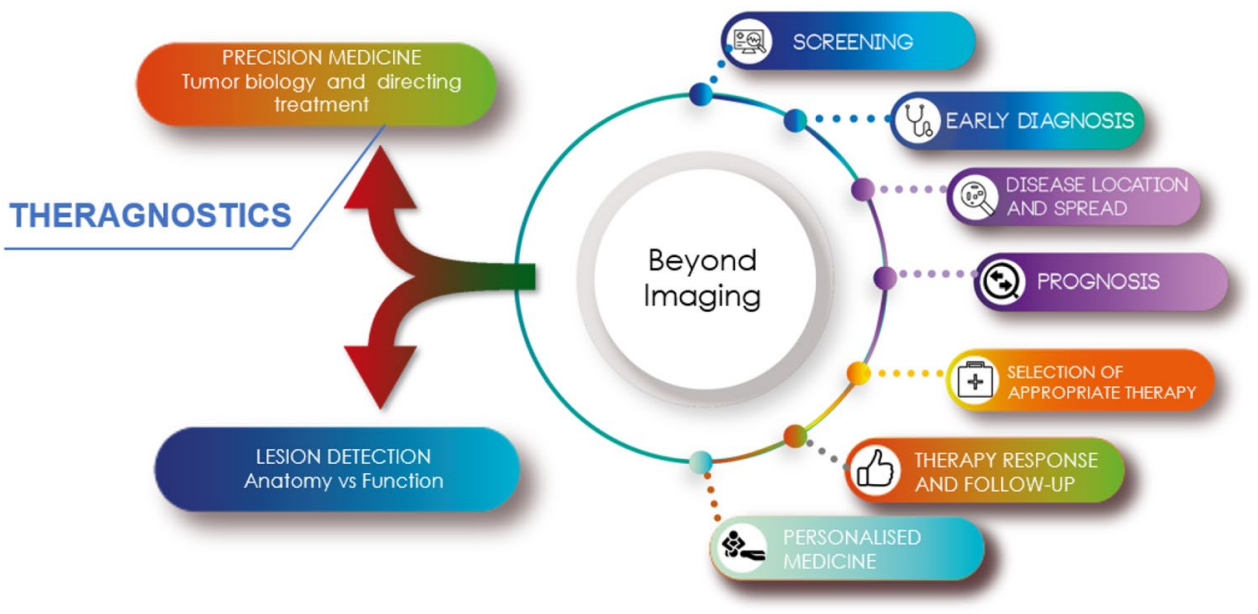


future for all [7]. Goal 3 focuses on ensuring healthy lives and promoting well-being for all at all ages. It is important to highlight two targets: target 3.4 addresses the challenges of reducing NCD premature mortality by $30 \%$ by 2030 and target 3.9 aims at strengthening the capacity of all countries, particularly developing countries, for early warning, risk reduction and management of national and global health risks.

To support national efforts to address the burden on NCDs, the 66th World Health Assembly endorsed the WHO Global Action Plan (GAP) for prevention and control of NCDs 2013-2020 (resolution WHA66.10) [8]. The GAP offers a paradigm shift by providing a roadmap and policy options menu for Member States, the WHO, other UN organizations, intergovernmental organizations, NGOs and the private sector. The hypothesis is that, if implemented collectively between 2013 and 2020, we will achieve nine voluntary global targets, including a relative $25 \%$ reduction in premature NCDs mortality by 2025. Target 8 is of pivotal importance to the medical imaging community. Its objective is to achieve $80 \%$ availability of affordable basic technologies and essential medicines, including generics required to treat NCDs in both public and private facilities. The problem is that medical imaging and nuclear medicine are not defined under target 8 . Nevertheless, there is an opportunity to include medical images as affordable or basic technologies, for which the professional community must participate in decision-making processes.

It is fundamental to advice the health authorities of the importance of including medical imaging and nuclear medicine as part of the policies, strategies, and action plans for health technologies and the national health plan.

Author contributions All authors contributed equally to the paper.

\section{Compliance with ethical standards}

Conflict of interest The authors declare that they have no conflict of interest.

Research involving human and animal rights This article does not contain any studies with human participants or animals performed by any of the authors.

Informed consent For this type of study, informed consent is not required.

\section{References}

1. World Bank Group. New Country Classifications by Income Level: 2019-2020. https://blogs.worldbank.org/opendata/newcountry-classifications-income-level-2019-2020

2. NUMDAB, NUclear Medicine DAtaBase. https://humanhealt h.iaea.org/HHW/NuclearMedicine/NUMDAB/index.html

3. IMAGINE, the new IAEA Medical imAGIng and Nuclear mEdicine global resources database. https://humanhealth.iaea.org/ HHW/DBStatistics/IMAGINE.html

4. Paez D, Becic T, Bhonsle U, Jalilian AR, Nuñez-Miller R, Osso JA Jr (2016) Current status of nuclear medicine practice in the Middle East. Semin Nucl Med 46(4):265-272

5. International Atomic Energy Agency, Planning a Clinical PET Centre, Human Health Series No. 11, IAEA, Vienna (2010)

6. Páez D, Orellana P, Gutiérrez C, Ramirez R, Mut F, Torres L (2015) Current status of nuclear medicine practice in Latin America and the Caribbean. J Nucl Med 56(10):1629-1634

7. United National Sustainable Development Goals. https://susta inabledevelopment.un.org

8. World Health Organization. Global Action Plan for Healthy Lives and Well-being for All. https://www.who.int/sdg/global-actio n-plan

Publisher's Note Springer Nature remains neutral with regard to jurisdictional claims in published maps and institutional affiliations. 